\title{
Temporary Segmental Renal Artery Occlusion Using Reverse Phase Polymer for Bloodless Robotic Partial Nephrectomy
}

\author{
Alireza Moinzadeh ${ }^{\star}, \dagger$, Sebastian Flacke, John A. Libertino, John Merhige $\ddagger$, Jean-Marie \\ Voge ${ }^{\ddagger}$, Katy Lyall $\dagger$, Curtis W. Bakal, and Peter N. Madras ${ }^{\ddagger}$ \\ From the Lahey Clinic, Burlington and Pluromed, Inc., Woburn, Massachusetts, and Intuitive \\ Surgical, Inc., Sunnyvale, California
}

\begin{abstract}
Purpose-Renal vascular clamping with ensuing warm ischemia is typically needed during robotic or laparoscopic partial nephrectomy. We developed a technique for angiographic delivery of the novel intra-arterial reverse thermoplastic polymer LeGoo-XL $\mathrm{XL}^{\mathrm{TM}}$ that allows temporary selective vascular occlusion with normal perfusion of the remaining kidney.

Materials and Methods-Eight pigs underwent a total of 16 selective angiographic occlusions of the lower pole segmental artery using gel polymer. The technical feasibility of 2 hemostatic techniques, perfusion hemostasis and local plug formation, was assessed in 4 pigs each. Selective ischemia time was recorded and the vascular occlusion site was noted radiographically and laparoscopically. The feasibility of reversing the polymer from solid back to liquid state to allow reperfusion was determined. Pathological analysis of the kidney was completed in these acute model pigs. In the last 2 cases lower pole robotic partial nephrectomy was done using the da Vinci ${ }^{\circledR}$ surgical system.
\end{abstract}

Results-Selective lower pole ischemia was achieved in all 8 cases. Perfusion hemostasis yielded an inconsistent duration of occlusion (zero to greater than 60 minutes). Vascular occlusion time using local plug formation was more reliable (17 to 30 minutes) with consistent ability to reverse the plug to liquid state by cold saline flush. Two lower pole robotic partial nephrectomies were completed with minimal blood loss.

Conclusions-We developed a reliable technique of angiographic delivery of gel polymer for temporary vascular occlusion of selective renal artery branches using local plug formation. Ongoing studies are under way to assess technique consistency and the long-term effects of the polymer.

\section{Keywords}

kidney; nephrectomy; robotics; laparoscopy; polymers

The incidence of renal malignancies has increased dramatically in the last 30 years. ${ }^{1}$

Concurrently the technical feasibility and long-term oncological success of nephron sparing

\footnotetext{
${ }^{\dagger}$ Financial interest and/or other relationship with Intuitive Surgical.

FFinancial interest and/or other relationship with Pluromed.

Copyright () 2009 by American Urological Association

*Correspondence and requests for reprints: Robotic Surgery, Institute of Urology, Urologic Oncology, Lahey Clinic, 41 Mall Rd., Burlington, Massachusetts 01805 (telephone: 781-744-8334; FAX: 781-744-5429; moinza00@lahey.org).

Study received institutional animal care and use committee approval.

Study contents are solely the responsibility of the authors and do not necessarily represent the official views of National Institutes of Health, National Institute for Diabetes and Digestive and Kidney Diseases.
} 
surgery has been demonstrated. ${ }^{2}$ Improvements in minimally invasive equipment and techniques during the last decade have allowed laparoscopic partial nephrectomy to emerge as a viable alternative to open surgery. ${ }^{3}$ However, the technical demands of the procedure and potential issues related to warm ischemia have hindered widespread acceptance.

The novel gel polymer LeGoo-XL is a nontoxic, nonionic polymer with rapid reverse thermosensitive properties. The polymer exists as a liquid at cold or room temperature and rapidly transitions to a viscous gel as it approaches body temperature. It reverts to a liquid with cooling and cannot return to a gel after dissolving. The polymer is excreted in urine. Based on early development studies we realized that LeGoo-XL injection may be accomplished by 1 of 2 techniques. When injected into arteries rapidly at room temperature, the polymer immediately forms a LP that blocks blood flow at or near the injection site. Plug reversal is almost instantaneous with cold saline perfusion. In contrast, renal flow interruption may also be accomplished by infusing the polymer slowly at lower concentrations and temperatures, allowing flow deeper into the end arteries and arterioles, forming longer gel columns that propagate back toward the injection site. We termed this injection technique PH.

We assessed the feasibility of controlling the extent and duration of renal artery occlusion by delivering gel polymer by these 2 delivery techniques. Also, since angiographically placed catheters are readily advanced into renal artery branches, we postulated that it would be possible to selectively interrupt flow to only a renal segment targeted for resection while allowing the untargeted kidney portion to retain normal flow. During the flow interruption period we examined the feasibility of performing robotic assisted laparoscopic partial nephrectomy.

\section{METHODS}

The study was approved by the institutional animal care and use committee. Eight farm pigs underwent general anesthesia, followed by angiographic access to the lower segmental branch of the renal artery. Experiments were divided into 2 groups. In 4 group 1 pigs blood flow was interrupted by PH and in 4 in group 2 a LP technique was used. In group 1 all gel polymer injections were performed slowly at approximately $1 \mathrm{cc}$ per second for 3 seconds, and at lower temperatures and concentrations to achieve full $\mathrm{PH}$. A relatively dilute solution (15\% to $17 \%$ gel polymer) yielding lower viscosity permitted further polymer penetration into more distal, smaller arteries. In group 2 with LP a localized gel polymer vascular plug of about $2 \mathrm{~cm}$ was formed in the arterial region immediately distal to the catheter tip. For this purpose a more concentrated solution ( $20 \%$ gel polymer) was delivered rapidly. The increased concentration gave the plug increased viscosity and extended the flow interruption duration. In all cases the lower kidney pole was the target for flow interruption and resection. The groups were compared for the extent and duration of flow interruption, and the ability to promptly return flow by iced saline infusion. Injection volume and time, and polymer concentration and temperature used were recorded. When lower pole flow interruption appeared complete, we assessed the feasibility of using the da Vinci surgical robot for partial nephrectomy.

\section{Angiographic Techniques}

Angiography was accomplished under $\mathrm{C}$ arm fluoroscopy using a $7 \mathrm{Fr}$ guiding catheter and $5 \mathrm{Fr}$ injection catheter (Renal Access Cobra Catheter, Cook, Bloomington, Indiana). The catheter was advanced to the lower pole segmental branch of the renal artery using standard angiographic techniques. Before gel polymer injection renal angiography was performed using hand injection. In the first 2 pigs tantalum particles were suspended in gel polymer to allow visualization of the forming plug but visualization was not optimal. In the last 6 experiments the contrast agent Omnipaque ${ }^{\mathrm{TM}} 300$ was mixed with the gel polymer solution. 
In all pigs the lower pole branch of the renal artery was targeted for occlusion. In 7 cases the renal artery bifurcated into an upper and a lower pole major branch while in 1 the bifurcation appeared to be more anterior to posterior and tertiary branch injection was required to target the lower pole. The initial pig experiment was performed in open fashion to enable visual and Doppler observation of the kidney during gel polymer infusion. All subsequent experiments were performed using the da Vinci robotic system. In the first 4 experiments full PH of the targeted region was achieved and in the final 4 gel polymer was deposited as a localized plug approximately $2 \mathrm{~cm}$ long exactly in the lower segmental branch of the renal artery, blocking flow to the distal vessels but not filling them with polymer. The ability to dissolve LP or PH was studied by infusing cold saline through the angiocatheter.

\section{Robotic Dissection and Partial Nephrectomy}

Robot assisted partial nephrectomy was performed using 5-port transperitoneal access. The da Vinci Si HD ${ }^{\mathrm{TM}}$ robotic system was docked. The peritoneal reflection over the kidney was removed to allow future robotic lower pole partial nephrectomy after flow to the lower pole was interrupted. The robotic camera was necessary for visual inspection of the kidney as needed.

After satisfactory visual (laparoscopic) and angiographic confirmation of arterial occlusion the robot was redocked and lower pole partial nephrectomy was performed. At the completion of resection the renal tissue was approximated over a hemostatic bolster vs primary capsular closure. Time to return of blood flow was recorded. The remnant kidney was analyzed with hematoxylin and eosin staining, and pathological analysis.

\section{RESULTS}

Mean weight of the 8 pigs was 133 pounds (range 107 to 155). Estimated blood loss in 4 robotic partial nephrectomy cases was less than $50 \mathrm{cc}$. The table lists the concentration of polymer used, injection volume and temperature, occlusion time and comments on each injection. In pig 1 surgery was performed in open fashion. A demarcation line was immediately seen after vascular occlusion, corresponding exactly to the distribution anticipated from the angiogram. In all subsequent animals this consistent relationship was noted between angiographic appearance and surface demarcation. While this was expected, it verified that perfusion to a specified kidney segment could be turned off in a manner predicted almost perfectly by angiogram, while perfusion to the remaining kidney portion remained unimpaired. Figure 1 shows angiograms and corresponding gross images of the kidney before and after intrarenal branch occlusion.

To achieve the localized arterial plug previous developmental studies using a circulation model showed that more concentrated gel polymer (20\%) was required. This resulted in increased viscosity of the liquid solution and a tendency for some gelation to occur in the angiographic catheter. To achieve consistent polymer injections a manually powered, screw driven injector was used instead of simple hand injection in pigs 5 to 8 . Plug formation and growth were observed under fluoroscopy and accurately controlled with the injector.

The table shows that occlusion time in pigs that underwent PH varied highly, and remained inconsistent and unpredictable even when the catheter tip was in the branch targeting only the lower kidney pole. The ability to resume flow at will by dissolving the polymer with iced saline was inconsistent. Since these injections were done by hand, gel polymer concentration could not exceed $17 \%$ before viscosity became high enough to preclude hand injection. However, examination in pigs 5 to 8 showed that it was possible to produce a localized gel polymer plug with great consistency. In pig 5 we still used 17\% polymer, which did not produce a robust plug. However, $20 \%$ gel polymer injected using the screw injector delivered exactly 0.6 to 0.8 
$\mathrm{ml}$ into the lower segmental branch of the renal artery, producing a completely reproducible, reliable vascular plug that lasted 30 minutes and was readily reversed by iced saline infusion. In the final 2 experiments robotic partial nephrectomy of the lower pole was done with minimal blood loss. In these cases at the completion of resection an infusion of $50 \mathrm{ml}$ iced saline through the angiographic catheter ensured rapid, complete plug dissolution with the return of flow (fig. 2). The remnant kidney was examined primarily to assess polymer residue or evidence of local infarction, which were not visualized at pathological analysis.

\section{DISCUSSION}

Renal function preservation, and complete removal of tumor or tumor kill during ablation is the main goal of nephron sparing surgery. Although several competing probe ablative therapies have emerged for small renal masses, partial nephrectomy remains the standard for comparison. The ideal partial nephrectomy would consist of tumor removal without warm or cold ischemia. A recent retrospective study of more than 500 open partial nephrectomies performed in a solitary kidney at 2 institutions revealed that renal artery clamping vs nonclamping led to a statistically significant increase in urine leak, and acute and chronic renal failure. ${ }^{4}$ The group conclude that warm ischemia time should be minimized.

Contemporary techniques for laparoscopic partial nephrectomy typically do not avoid warm ischemia. 5 Proposed techniques to minimize any associated renal injury are clamping avoidance, ${ }^{6}$ renal cooling with ice slush, ${ }^{7}$ renal artery cold perfusion ${ }^{8}$ or transureteral retrograde renal hypothermia ${ }^{9}$ and various forms of energy based tumor resection, including bipolar, ${ }^{10}$ laser $^{11}$ and radio frequency. ${ }^{12}$ During open surgery several nonrenal artery clamping techniques have been described that obviate the need for total renal ischemia. ${ }^{13,14}$

Renal cooling with ice slush (nonclamping) adequately decreases core parenchymal temperature but it is tedious and has largely been abandoned. Cooling through the ureter or renal artery with $4 \mathrm{C}$ irrigation may not be sufficient to achieve a major benefit. Recently earlier unclamping by Nguyen and Gill achieved a significant decrease in warm ischemia time to approximately 14 minutes. ${ }^{15}$ However, they had experience with more than 600 laparoscopic partial nephrectomies and extrapolating their results to less experienced surgeons may be unrealistic.

One of the earliest partial nephrectomy series without hilar clamping was reported by Guillonneau et al. ${ }^{6}$ They compared clamped vs unclamped laparoscopic partial nephrectomy and noted significantly increased blood loss (270 vs $708 \mathrm{cc}$ ). Given the technical challenges, Ultracision $®$ and bipolar based tumor resections have not been widely adopted except for small exophytic renal masses. Other energy based tumor resections are feasible in animal models but have not provided significant clinical results. Energy based resection may be ideal for small exophytic tumors but several potential problems exist for larger or more parenchymal masses. Tumor resection in a perfused kidney leads to increased blood loss, which may increase the positive margin rate. Energy based tumor resection may make identifying pathological margins more challenging, given tissue char. The repair of collecting system entry may be impaired, given thermal energy tissue disruption, which ultimately increases the risk of urine leak.

The purified nonionic poloxamer gel polymer used in this study was previously evaluated for temporary coronary artery occlusion in the porcine model during coronary artery bypass grafting with encouraging early results. ${ }^{16}$ Excellent preliminary clinical results were reported in a small series of patients undergoing coronary artery bypass grafting (10) and peripheral vascular bypass (3) using LeGoo $^{\mathrm{TM}}$, which has a composition similar to that of LeGoo-XL but is tailored for coronary and peripheral temporary occlusion. ${ }^{17}$ 
In a developmental study we evaluated several variables related to the polymer ability to completely occlude the renal artery (unpublished data). This series was done in flow models and verified in the porcine model in 7 animals. The variables were polymer concentration, polymer temperature at injection, and injection volume and rate. Based on this study we hypothesized that the polymer unique thermosensitive property of liquid state at low temperatures and gel state at body temperature would allow temporary renal vascular occlusion during partial nephrectomy.

Several advantages exist for the selective arterial occlusion techniques used in this study. Renal vascular dissection is not necessary, minimizing the risk of inadvertent renal vascular injury. Avoiding renal artery clamping in patients with renal atherosclerosis would decrease the risk of plaque rupture and distal shower emboli. The angiographic local plug injection technique allowed consistent targeted segmental occlusion. Selective temporary occlusion to the kidney portion containing the tumor allowed tumor resection in a bloodless field while retaining normal perfusion of the uninvolved kidney portion.

Some shortcomings of our current feasibility study exist. Without a chronic survival model long-term pathological data on the polymer tissue effect are uncertain, although immediate pathological examination revealed no abnormality in the remnant kidney. Interventional radiological expertise is required for femoral access and selective arterial catheterization. This approach carries its own risk, although it is low. A recent review of 19,826 consecutive cerebral angiographies showed local access hematoma in $4.2 \%$ with surgery required in $0.03 \%{ }^{18}$ Thrombosis and infection developed in $0.05 \%$ and $0.01 \%$ of cases, respectively. Although it is technically feasible for lower pole partial nephrectomy, larger tumors deriving the blood supply from several segmental branches may not be suitable for this technique or may require the occlusion of multiple segmental arteries. Finally, selective occlusion requires a radiographic contrast agent, which may be harmful to the ipsilateral and the contralateral kidney when used in large volumes.

\title{
CONCLUSIONS
}

Temporary targeted renal segment occlusion is feasible with LeGoo-XL gel polymer. Our experience culminated in temporary selective arterial occlusion in the porcine model to perform robotic partial nephrectomy of the lower pole with essentially no blood loss while flow continued uninterrupted to the upper pole. Further chronic survival studies are under way to determine any long-term pathological and functional renal or systemic complications associated with LeGoo-XL use.

\section{Abbreviations and Acronyms}

\author{
LP local plug formation \\ $\mathrm{PH} \quad$ perfusion hemostasis
}

\section{Acknowledgments}

Michael Burns, Intuitive Surgical, Inc., and Phil Codyer, Robert Lichstein and Steven Binzel, Lahey Clinic assisted with the study. Intuitive Surgical, Inc. provided the robot.

Supported by Grant 1R43DK079481-01 from the National Institutes of Health, National Institute for Diabetes and Digestive and Kidney Diseases awarding component and Lahey Clinic Robert E. Wise Foundation.

\section{REFERENCES}

1. Seer Cancer Statistics Review 1975-2005. Bethesda: National Cancer Institute; 2005. 
2. Fergany AF, Hafez KS, Novick AC. Long-term results of nephron sparing surgery for localized renal cell carcinoma: 10-year followup. J Urol 2000;163:442. [PubMed: 10647650]

3. Lane BR, Gill IS. 5-Year outcomes of laparoscopic partial nephrectomy. J Urol 2007;177:70. [PubMed: 17162003]

4. Thompson RH, Frank I, Lohse CM, et al. The impact of ischemia time during open nephron sparing surgery on solitary kidneys: a multi-institutional study. J Urol 2007;177:471. [PubMed: 17222613]

5. Haber GP, Gill IS. Laparoscopic partial nephrectomy: contemporary technique and outcomes. Eur Urol 2006;49:660. [PubMed: 16504373]

6. Guillonneau B, Bermudez H, Gholami S, et al. Laparoscopic partial nephrectomy for renal tumor: single center experience comparing clamping and no clamping techniques of the renal vasculature. $\mathrm{J}$ Urol 2003;169:483. [PubMed: 12544293]

7. Gill IS, Abreu SC, Desai MM, et al. Laparoscopic ice slush renal hypothermia for partial nephrectomy: the initial experience. J Urol 2003;170:52. [PubMed: 12796643]

8. Janetschek G, Abdelmaksoud A, Bagheri F, et al. Laparoscopic partial nephrectomy in cold ischemia: renal artery perfusion. J Urol 2004;171:68. [PubMed: 14665846]

9. Crain DS, Spencer CR, Favata MA, et al. Transureteral saline perfusion to obtain renal hypothermia: potential application in laparoscopic partial nephrectomy. JSLS 2004;8:217. [PubMed: 15347107]

10. Moinzadeh A, Hasan W, Spaliviero M, et al. Water jet assisted laparoscopic partial nephrectomy without hilar clamping in the calf model. J Urol 2005;174:317. [PubMed: 15947682]

11. Moinzadeh A, Gill IS, Rubenstein M, et al. Potassium-titanyl-phosphate laser laparoscopic partial nephrectomy without hilar clamping in the survival calf model. J Urol 2005;174:1110. [PubMed: 16094075]

12. Urena R, Mendez F, Woods M, et al. Laparoscopic partial nephrectomy of solid renal masses without hilar clamping using a monopolar radio frequency device. J Urol 2004;171:1054. [PubMed: 14767269]

13. Smith G, Cohen M, Kurteva T, et al. Non-clamped, non-ischemic partial nephrectomy in patients with compromised preoperative renal function or with a solitary kidney. J Urol 2008; 179:377. abstract 1096.

14. Thompson RH, Leibovich BC, Lohse CM, et al. Complications of contemporary open nephron sparing surgery: a single institution experience. J Urol 2005;174:855. [PubMed: 16093969]

15. Nguyen MM, Gill IS. Halving ischemia time during laparoscopic partial nephrectomy. J Urol 2008;179:627. [PubMed: 18082215]

16. Aubin MC, Bouchot O, Carrier M, et al. Temporary internal thoracic artery occlusion during offpump coronary artery bypass grafting with the new poloxamer P407 does not cause endothelial dysfunction. J Thorac Cardiovasc Surg 2006;132:685. [PubMed: 16935129]

17. Bouchot, O.; Steinmetz, E.; Berne, J., et al. Novel use of a gel for temporary vascular occlusion during surgery of small vessels in human: preliminary results; Presented at meeting of International Society of Minimally Invasive Cardiothoracic Surgery; Boston, Massachusetts: 2008 Jun 11-14.

18. Kaufmann TJ, Huston J 3rd, Mandrekar JN, et al. Complications of diagnostic cerebral angiography: evaluation of 19,826 consecutive patients. Radiology 2007;243:812. [PubMed: 17517935] 

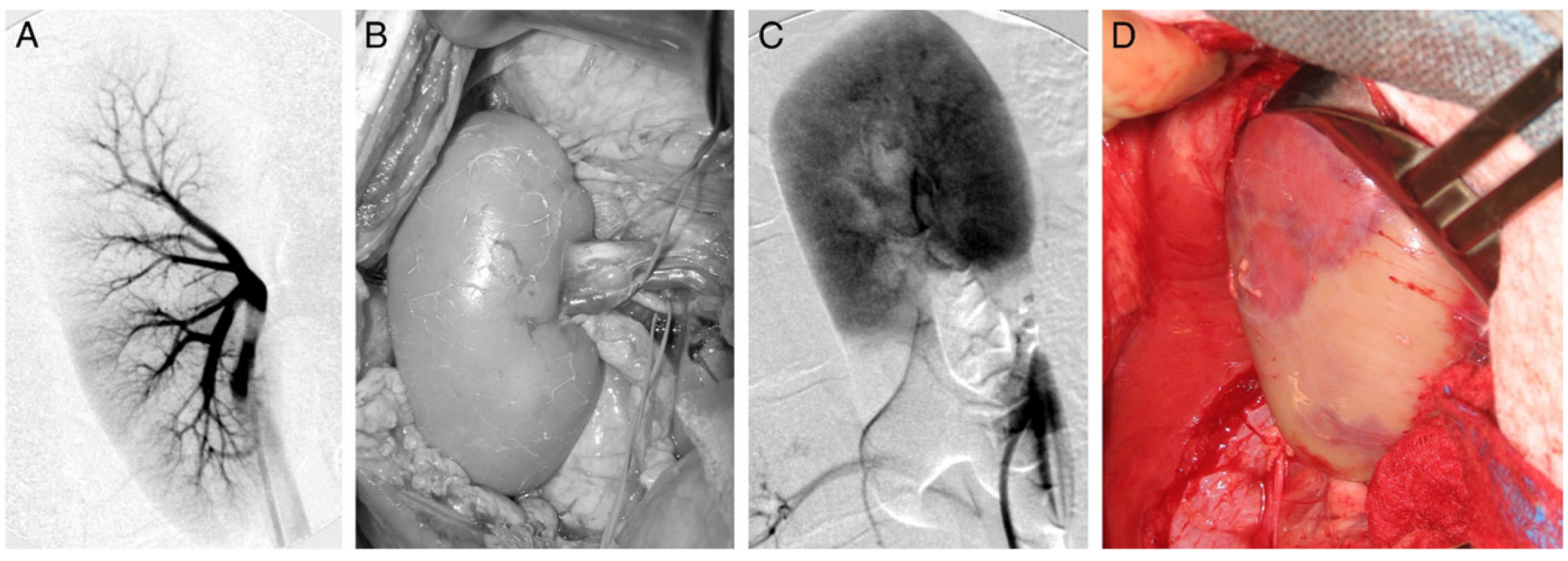

Figure 1.

$A$ and $B$, pre-injection baseline. $A$, arteriogram shows early phase with contrast material identifying main renal artery and 2 main branches per pole. $B$, direct gross visualization correlates with arteriogram. $C$ and $D, 2$ minutes after injection into renal artery lower segmental branch. $C$, arteriogram shows lower pole segmental occlusion. At small area of lower kidney blush is clearly provided from upper branch by small vessel. Note no evidence of flow to any other part of lower kidney portion. Circulation to lower pole was completely interrupted but flow to upper kidney portion was normal. $D$, gross image shows blanched lower pole and pink upper pole, correlating well with arteriography. 


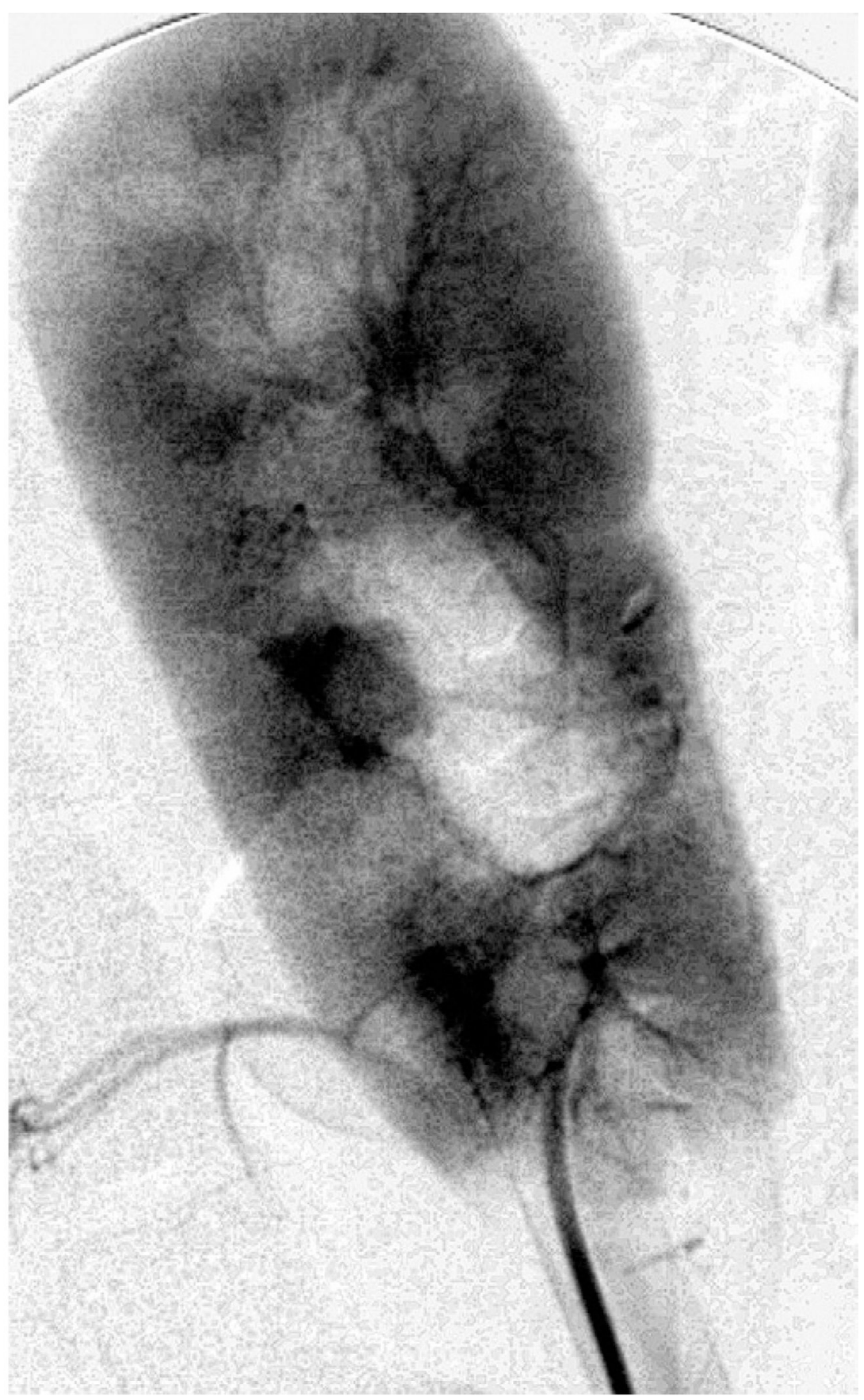

Figure 2.

After robotic resection of lower $20 \%$ of kidney arteriogram reveals restored flow in lower segmental branch with contrast material reaching into small arterioles and full renal blush. 


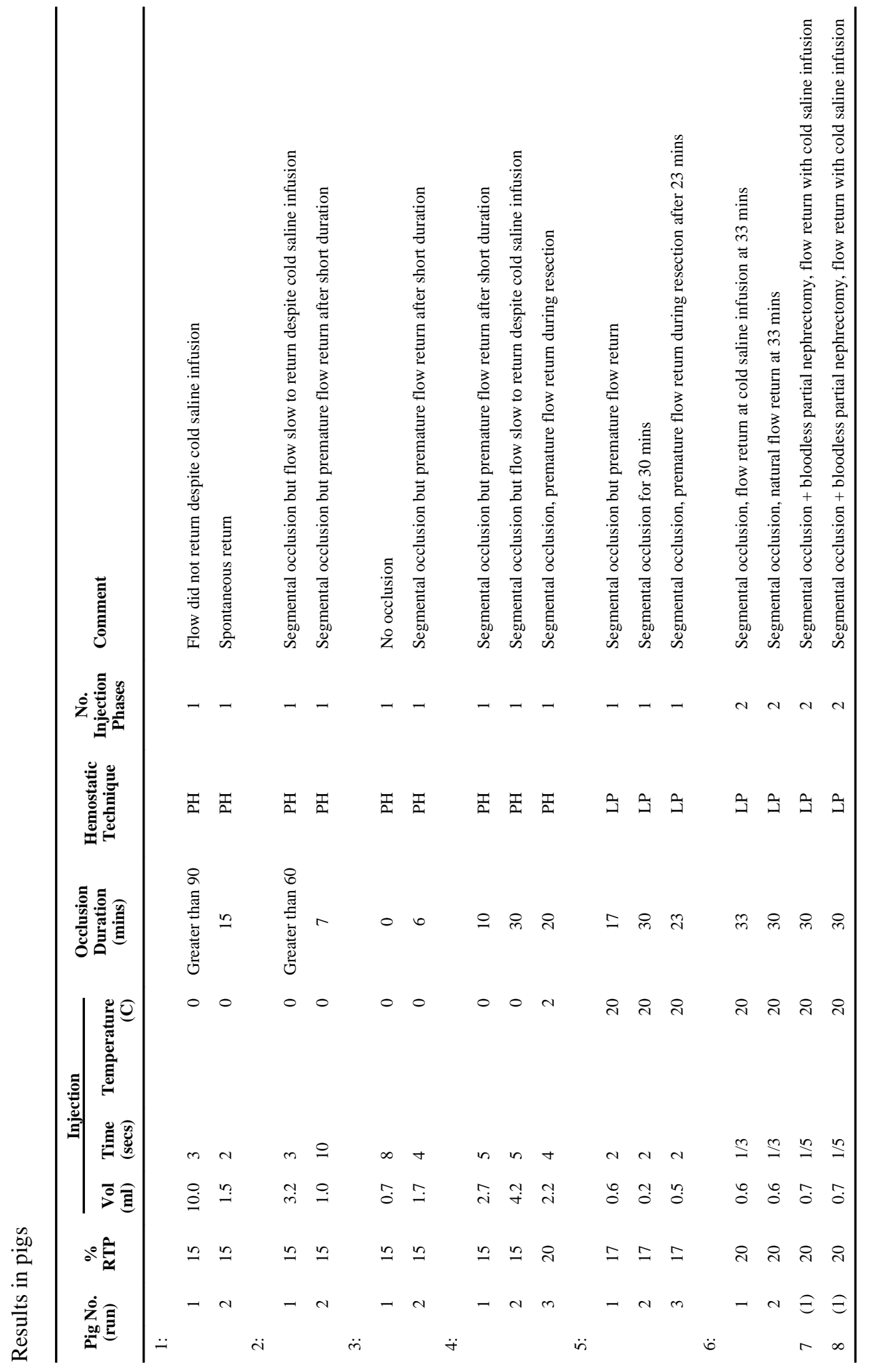

J Urol. Author manuscript; available in PMC 2010 September 20. 